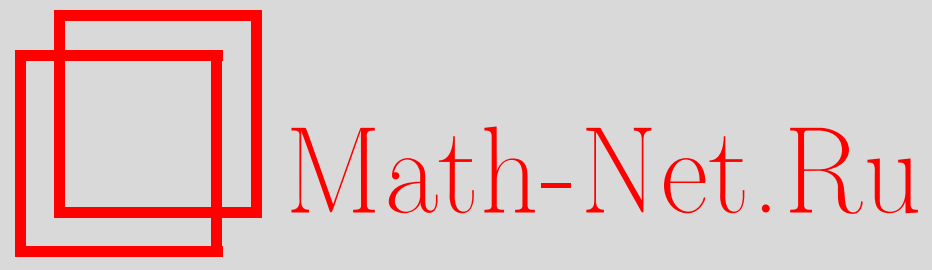

М. М. Бузмакова, Перколяция вытянутых эллипсоидов вращения в континууме, Вестн. Сам. гос. техн. ун-та. Сер. Физ.-мат. науки, 2012, выпуск 4(), 146 153

DOI: https://doi.org/10.14498/vsgtu1095

Использование Общероссийского математического портала Math-Net.Ru подразумевает, что вы прочитали и согласны с пользовательским соглашением

http://www.mathnet.ru/rus/agreement

Параметры загрузки:

IP : 54.209 .52 .79

26 апреля 2023 г., $17: 43: 09$

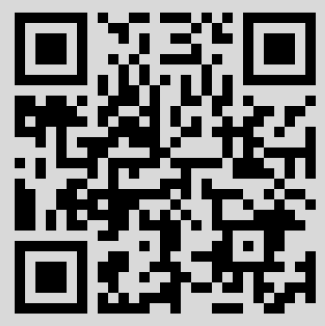


Вестн. Сам. гос. техн. ун-та. Сер. Физ.-мат. науки. 2012. № 4 (29). С. $146-153$

УДК 519.68:[5/6+3]+004.94+544.015.4+544.022.822

\title{
ПЕРКОЛЯЦИЯ ВЫТЯНУТЫХ ЭЛЛИПСОИДОВ ВРАЩЕНИЯ В КОНТИНУУМЕ
}

\author{
М. М. Бузмакова \\ Астраханский государственный университет, \\ 414056, Россия, Астрахань, ул. Татищева, 20 а. \\ E-mail: mariya_nazarova@mail.ru
}

\begin{abstract}
Исследована континуальная перколяция жёстких вытянутых эллипсоидов вращения с проницаемой оболочкой, которая может служить моделъю фазового перехода золь-гель. Эллипсоидъ случайным образом помещаются в куб. Для каждого набора параметров проводится 100 испытаний. Для каждого испытания главной задачей является нахождение перколяционного кластера. Та доля заполнения системы, при которой вероятность возникновения перколячионного кластера равна 0,5, называется порогом перколяиии. Значение порога перколяиии соответствует точке геля. Получена зависимость значения порога перколяции от толщины проницаемой оболочки и аспектного отношения эллипсоида. Кроме порога перколячии рассчитаны другие характеристики модели: распределение кластеров по размерам, средний размер кластера, мощность и фрактальная размерность перколяционного кластера, среднее значение и распределение соседей элемента, критические показатели.
\end{abstract}

Ключевые слова: перколяиия, гелеобразование, фазовые переходы.

Введение. При высыхании капель биологических жидкостей происходит множество физических, биологических, биофизических, биохимических и физико-химических процессов, изучение которых на сегодняшний день представляет актуальную задачу. Одним из таких процессов является фазовый переход золь-гель белка альбумина, то есть переход из жидкой дисперсной среды в среду со структурой, придающей ей свойства твердого тела. Увеличение концентрации дисперсной фазы (в нашем случае молекул альбумина) приводит к появлению контактов между частицами и началу структурирования - гелеобразования.

Данная работа посвящена моделированию процесса гелеобразования белка альбумина в процессе дегидратации биологических жидкостей. При моделировании используются методы теории перколяции. Предложена модель континуальной перколяции жёстких вытянутых эллипсоидов вращения с проницаемыми оболочками. Эллипсоиды выступают в роли молекул альбумина, вероятность молекулярного докинга молекул характеризуется толщиной проницаемой оболочки эллипсоида. Порог перколяции соответствует точке геля (критическая концентрация молекул альбумина в водном растворе).

Теория перколяции изучает образование связанных объектов в неупорядоченной среде. С помощью теории перколяции описаны многие физические, химические и другие процессы $[1,2]$. Известны математические модели процесса гелеобразования, основанные на методах теории перколяции [3-5]. Используются решёточные и континуальные модели. В решёточных моделях занятые узлы рассматривают в качестве молекул растворенного вещества, а пустые-в качестве молекул растворителя. Однако в большинстве случаев

Мария Михайловна Бузмакова, ассистент, каф. прикладной математики и информатики. 
решёточные модели оказываются слишком упрощенными для описания реальных систем, так как, во-первых, молекулы растворенного вещества, как правило, не являются точечными объектами, во-вторых, координаты молекул в реальных системах являются непрерывными, а не дискретными. Исходя из этого требуется изучить процесс гелеобразования с помощью моделирования континуальной перколяционной системы.

В настоящей работе предлагается исследование перколяции эллипсоидов в пространстве. Континуальная перколяция эллипсоидов изучалась и ранее [69]. Новизна настоящего исследования заключается в том, что исследуются вытянутые жёсткие эллипсоиды с проницаемыми оболочками, пересечение которых обеспечивает наличие связи между эллипсоидами.

1. Постановка задачи. Основной целью моделирования является выявление зависимости порога перколяции от толщины проницаемой оболочки и аспектного отношения эллипсоида. Также актуально определение других основных характеристик системы, таких как распределение кластеров по размерам, средний размер кластера, мощность и фрактальная размерность перколяционного кластера, критические показатели, среднее количество соседей эллипсоида $B_{c}$.

Эллипсоид - поверхность второго порядка, полученная деформацией сферы вдоль трёх взаимно перпендикулярных осей. Эллипсоид вращения - эллипсоид, две полуоси которого равны и называются радиусом вращения. Аспектным отношением эллипсоида вращения является отношение большей полуоси к радиусу вращения. Как было отмечено выше, в задаче рассмотрены вытянутые эллипсоиды. Уравнение эллипсоида вращения имеет следующий вид:

$$
\frac{\left(x-x_{0}\right)^{2} \cos ^{2} \alpha \cos ^{2} \delta}{r^{2}}+\frac{\left(y-y_{0}\right)^{2} \cos ^{2} \alpha \sin ^{2} \delta}{r^{2}}+\frac{\left(z-z_{0}\right)^{2} \sin ^{2} \alpha}{(r k)^{2}}=1,
$$

где $x_{0}, y_{0}, z_{0}$ - координаты центра, $\alpha, \delta$ - углы наклона и поворота соответственно; $r$ - радиус вращения; $k$ - величина аспектного отношения.

Для достижения поставленной цели была создана компьютерная модель континуальной перколяции вытянутых жёстких эллипсоидов вращения (далее эллипсоидов) с проницаемыми оболочками. Эллипсоиды с радиусом $r$, аспектным отношением $k$ и толщиной проницаемой оболочки $d$ случайным образом помещаются в куб с линейным размером $L$. Два эллипсоида принадлежат одному кластеру в том случае, если их проницаемые оболочки пересекаются. В задаче ищется перколяционный кластер, то есть кластер, соединяющий нижний и верхний грани куба.

2. Методика моделирования. Для моделирования перколяции жёстких эллипсоидов с проницаемыми оболочками была создана программа на языке программирования C++. Входными данными программы являются: L линейный размер куба, r - радиус вращения, $\mathrm{k}$ - аспектное отношение эллипсоида, $\mathrm{h}$ - отношение толщины проницаемой оболочки к радиусу сферы, pmin - минимальное значение доли заполнения куба сферами, pmax - максимальное значение доли заполнения куба сферами, step-шаг изменения доли заполнения, ki - количество испытаний. При моделировании были использованы периодические граничные условия по всем трем направлениям. 
Моделирование проводилось методом Монте-Карло. Для генерации случайных чисел при упаковке эллипсоидов в куб применялся алгоритм «Вихрь Мерсенна» [10]. Для идентификации принадлежности эллипсоида к кластеру используется алгоритм Хошена-Копельмана [11], который был модифицирован под континуальную задачу. Модифицированный алгоритм ХошенаКопельмана отличается от классического тем, что в классическом алгоритме перебираются по порядку все слои решётки, а в модифицированном перебираются все объекты от 1 до $n$, где $n$ - число упакованных в куб эллипсоидов. Это даёт возможность работать алгоритму не на решётке, а в континууме. Для нахождения вероятности возникновения перколяции в системе необходимо проверить на каждом испытании, существует ли кластер, пронизывающий всю систему - перколяционный кластер. Перколяционный кластер ищется по направлению снизу вверх, то есть от объекта, находящегося в нижнем слое системы, к объекту, находящемуся в верхнем слое системы. Таких объектов может оказаться несколько, необходимо рассмотреть все. Для этого создаются два массива, в первый minz записываются все номера объектов, для которых вертикальная координата $z$ удовлетворяет условию $0 \leqslant z \leqslant(r+d)$, во второй maxz - номера объектов, для которых $L-(r+d) \leqslant z \leqslant L$. Далее для каждой пары объектов из первого и второго массивов, которые имеют одинаковые кластерные метки, определяется, существует ли непрерывный путь между ними с помощью «волнового алгоритма» [12]. Если такая пара объектов находится, то дальнейшие объекты уже не проверяются.

Эллипсоиды имеют жёсткую и проницаемую части. При упаковке эллипсоидов в куб необходима проверка на пересечение жёстких частей эллипсоидов, а при распределении эллипсоидов по кластерам необходима проверка на пересечение их проницаемых оболочек. Для нахождения пересечения жёстких частей двух эллипсоидов вращения, а также для их проницаемых оболочек в программе были созданы специальные функции. В обеих функциях используется уравнение эллипсоида (1). Входными данными служат номера двух эллипсоидов i и j, для которых производится проверка на пересекаемость. Для обоих эллипсоидов известны координаты центра, угол наклона и угол поворота, которые хранятся в массивах х, y, z, alfa, delta. Из всего куба с линейным размером $L$ выбирается параллелепипед с минимальными размерами такой, что в него входят оба рассматриваемые эллипсоида. Далее с шагом 0,05 перебираются все точки данного параллелепипеда. Для каждой точки проверяется, удовлетворяет ли она уравнениям і-того и ј-того эллипсоидов. Если такая находится, то эллипсоиды пересекаются. Выбор шага 0,05 является оптимальным в рамках данной перколяционной модели для заданных начальных параметров, т.к. при выборе меньшего шага (то есть рассмотрения большего количества точек) время расчетов значительно увеличивается, а полученные результаты практически совпадают. Функция нахождения пересечения проницаемых оболочек двух эллипсоидов аналогична предыдущей. В данном случае в уравнениях і-того и ј-того эллипсоидов учитывается проницаемая оболочка $d$ и параллелепипед также выбирается с учётом толщины проницаемой оболочки. Обе функции возвращают значение 1, если пересечение найдено, и 0, если пересечения нет.

Определение фрактальной размерности перколяционного кластера и критических показателей производится по стандартной методике [13]. 
Определение значения среднего количества соседей объекта $B_{c}[14]$ на пороге перколяции производится следующим образом. Сначала находим распределение количества соседей по объектам, то есть считаем количество объектов $N(k)$, имеющих $k$ соседей, где $0 \leqslant k \leqslant k_{\max }$. Среднее значение количества соседей объекта вычисляем по формуле $B_{c}=\frac{1}{n} \sum_{k=0}^{k_{\max }} k N(k)$, где $n-$ количество объектов.

3. Результаты моделирования. Произведены расчеты для модели со следующими параметрами: $L=15 ; r=0,5 ; k=1,2,3 ; h=0,1 ; 0,2 ; \ldots ; 1$ и $L=20 ; r=0,5 ; k=4 ; h=0,1 ; 0,2 ; \ldots ; 1$. Для каждых $k, h$ произведено 100 испытаний и определено значение порога перколяции по стандартной методике: определена вероятность возникновения перколяционного кластера $P(p)$; далее полученные результаты компьютерного эксперимента аппроксимируются функцией вида $P(p)=\left(1+\exp \left(-\left(p-p_{c}\right) a\right)\right)^{-1}$ (см., например, рис. 1); значение доли заполнения $p$, при которой вероятность возникновения перколяционного кластера равна 0,5, принимается за значение порога перколяции $p_{c}$. При аппроксимации учитываются ошибки проведенных измерений следующим образом. Для каждого значения вероятности возникновения перколяционного кластера найдено стандартное отклонение среднего $\sigma_{\bar{P}}=\sqrt{\frac{1}{N-1} \sum\left(P_{i}-\bar{P}\right)^{2}} / \sqrt{N}$. Далее с использованием критерия Стьюдента найден доверительный интервал $\bar{P} \pm t \sigma_{\bar{P}}$, в который с вероятностью $95 \%$ попадает наше значение вероятности возникновения перколяционного кластера, где $t=1,98-$ коэффициент Стьюдента.

Получена зависимость порога перколяции от толщины проницаемой оболочки эллипсоида при различных значениях аспектного отношения (рис. 2). Данные компьютерного моделирования аппроксимируются функциями вида

$$
p_{c}(h)=A \exp (-h / t)+b,
$$

коэффициенты $A, t, b$ представлены в табл. 1. На графике (см. рис. 2) видно, что для каждого аспектного отношения эллипсоида при уменьшении значения толщины проницаемой оболочки порог перколяции возрастает. Следует обратить внимание на то, что при увеличении аспектного отношения эллипсоида вращения значение порога перколяции увеличивается менее интенсивно,

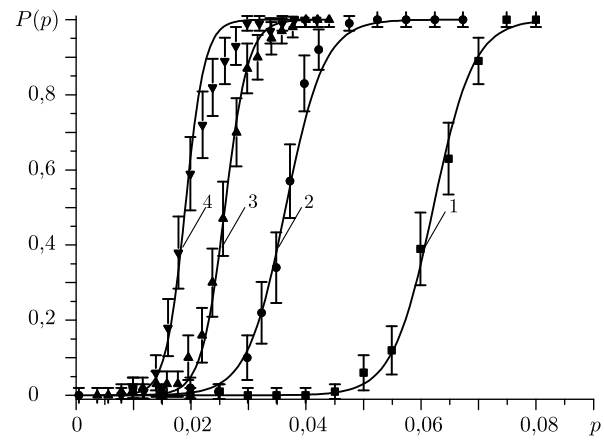

Рис. 1. Вероятность возникновения перколяционного кластера при $h=0,8: 1) k=$ $\left.=1, p_{c}=0,0618 \pm 0,0004 ; 2\right) k=2, p_{c}=$ $=0,0361 \pm 0,0002 ; 3) k=3, p_{c}=0,0258 \pm$ $0,0002$; 4$) k=4, p_{c}=0,0198 \pm 0,0002$

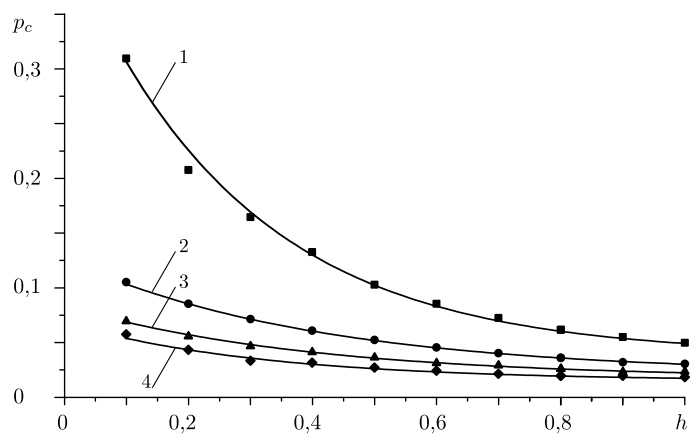

Рис. 2. Зависимость порога перколяции от толщины проницаемой оболочки эллипсоида при различных значениях аспектного отношения 


\section{Значения коэффициентов функций (2) при различных $k$}

\begin{tabular}{c|c|c|c}
\hline$k$ & $A$ & $t$ & $b$ \\
\hline 1 & $0,383 \pm 0,009$ & $0,28 \pm 0,02$ & $0,039 \pm 0,004$ \\
2 & $0,105 \pm 0,001$ & $0,40 \pm 0,01$ & $0,021 \pm 0,001$ \\
3 & $0,066 \pm 0,001$ & $0,39 \pm 0,02$ & $0,017 \pm 0,001$ \\
4 & $0,053 \pm 0,005$ & $0,32 \pm 0,05$ & $0,015 \pm 0,002$ \\
\hline
\end{tabular}

то есть если при аспектном отношении, равном единице, имеем изменение значения порога перколяции от $0,0462 \pm 0,0003$ до 0,3099 $\pm 0,0004$, то для аспектного отношения 4 - уже от 0,0165 $\pm 0,0003$ до 0,0585 $\pm 0,0009$. Таким образом, можно сделать вывод, что при увеличении аспектного отношения эллипсоида уменьшается влияние значения толщины проницаемой оболочки на значение порога перколяции. Исходя из этого можно предположить, что при каком-то большем значении аспектного отношения эллипсоида значение порога перколяции вообще не будет меняться при изменении толщины проницаемой оболочки либо изменится незначительно.

Кроме определения зависимости порога перколяции от толщины проницаемой оболочки были рассчитаны другие основные характеристики системы: распределение кластеров по размерам (определяет среднее количество кластеров, имеющих размер $s, 1 \leqslant s \leqslant s_{\max }, s_{\max }$ - максимальный размер кластера), средний размер кластера, мощность перколяционного кластера (вероятность того, что случайно выбранный элемент принадлежит перколяционному кластеру). Дополнительным подтверждением правильности полученных результатов можно считать характерное поведение среднего размера кластера $S(p)$ и мощности перколяционного кластера $P_{\infty}(p)$ [13] (рис. 3). Значение среднего размера кластера при приближении к порогу перколяции стремительно возрастает, а после порога перколяции стремительно убывает. Значение мощности перколяционного кластера при приближении к порогу перколяции стремительно возрастает, после порога перколяции возрастает менее интенсивно.

В настоящей работе определены критические показатели по стандартной методике [1]: $\gamma=1,63 \pm 0,15$ при $p>p_{c}, \gamma=1,97 \pm 0,17$ при $p<p_{c}$ и $\beta=$
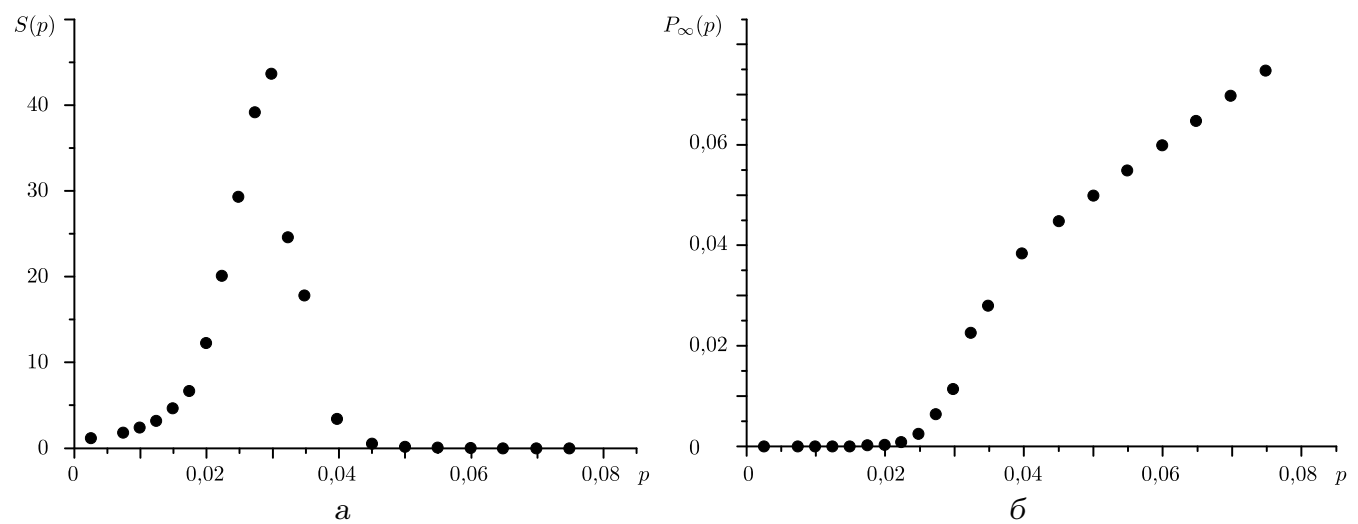

Рис. 3. Средний размер кластера (а) и мощность перколяционного кластера (б) при $L=15, h=1, k=2$ 
$=0,445 \pm 0,008$. Найденные значения равны в пределах погрешности ранее известным, что также подтверждает правильность полученных результатов.

Перколяционный кластер имеет фрактальную структуру. Представляет интерес определение значения фрактальной размерности перколяционного кластера на пороге перколяции. Для данной модели получены следующие значения фрактальной размерности перколяционного кластера: $D_{f}=2,74 \pm$ 0,05 для $k=4, h=0,8 ; D_{f}=2,79 \pm 0,07$ для $k=4, h=0,6$, которые в пределах погрешности совпадают между собой, совпадают с найденным значением работе [15] и совпадают с ранее известными [1]. Это также может служить подтверждением правильности полученных нами результатов.

Особый интерес для данной модели представляет нахождение значения среднего количества соседей у каждого эллипсоида $B_{c}$ (табл. 2 ) и распределение $B_{c}$ (рис. 4 ), поскольку никакие значения не были известны до получения наших результатов. Наблюдается общая закономерность для каждого аспектного отношения эллипсоида: при уменьшении значения толщины проницаемой оболочки уменьшается $B_{c}$. Причём для каждого аспектного отношения эллипсоида диапазоны изменения значения среднего количества соседей $B_{c}$ приблизительно совпадают (от 2,6 до 1,4).

Таблица 2

Среднее значение $B_{c}$ при различных $h$ и $k$

\begin{tabular}{c|c|c|c}
\hline \multicolumn{1}{c|}{$h$} & $k=2$ & $k=3$ & $k=4$ \\
\hline 1 & $2,54 \pm 0,01$ & $2,61 \pm 0,03$ & $2,58 \pm 0,03$ \\
0,9 & $2,47 \pm 0,01$ & $2,61 \pm 0,02$ & $2,51 \pm 0,02$ \\
0,8 & $2,37 \pm 0,01$ & $2,47 \pm 0,01$ & $2,48 \pm 0,02$ \\
0,7 & $2,30 \pm 0,01$ & $2,46 \pm 0,02$ & $2,37 \pm 0,03$ \\
0,6 & $2,24 \pm 0,01$ & $2,39 \pm 0,02$ & $2,33 \pm 0,03$ \\
0,5 & $2,21 \pm 0,01$ & $2,33 \pm 0,02$ & $2,24 \pm 0,05$ \\
0,4 & $2,08 \pm 0,01$ & $2,24 \pm 0,02$ & $2,21 \pm 0,03$ \\
0,3 & $1,99 \pm 0,01$ & $2,06 \pm 0,02$ & $1,82 \pm 0,02$ \\
0,2 & $1,89 \pm 0,01$ & $1,87 \pm 0,01$ & $1,71 \pm 0,03$ \\
0,1 & $1,60 \pm 0,01$ & $1,47 \pm 0,02$ & $1,38 \pm 0,05$ \\
\hline
\end{tabular}
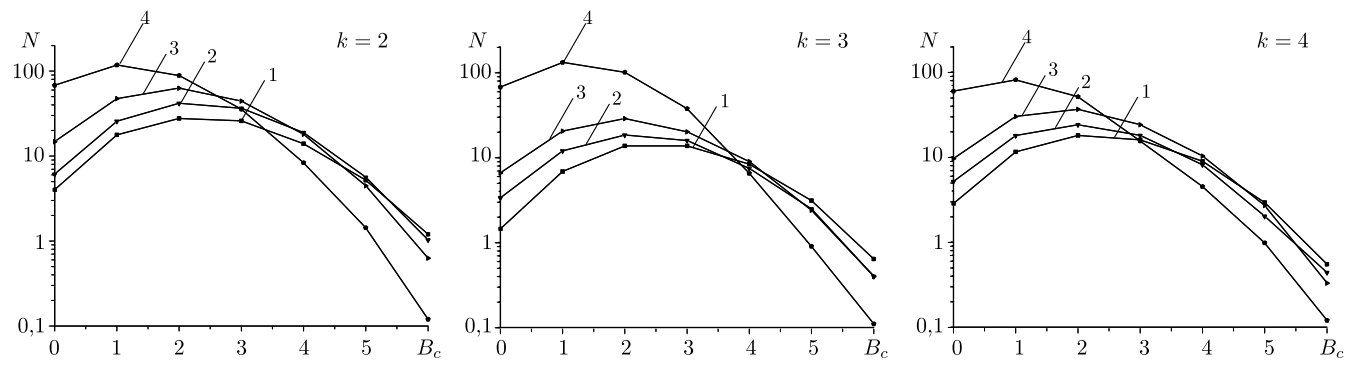

Рис. 4. Распределение количества эллипсоидов $N$, имеющих $B_{c}$ соседей на пороге перколяции при различных значениях $h$ и $k: 1-h=1 ; 2-h=0,7 ; 3-h=0,4 ; 4-h=0,1$

Заключение. Исследована континуальная перколяция жёстких вытянутых эллипсоидов вращения с проницаемыми оболочками. Получена зависимость порога перколяции от толщины проницаемой оболочки при значениях аспектного отношения $1,2,3,4$. Также определены такие характеристики 
системы, как средний размер кластера, распределение кластеров по размерам, мощность и фрактальная размерность перколяционного кластера. Кроме того, определены критические показатели $\gamma, \beta$. Впервые определены распределение эллипсоидов по соседям и среднее число соседей эллипсоида $B_{c}$. С точки зрения приложений следует отметить, что полученные результаты позволяют оценить концентрацию молекул альбумина в водном растворе, при которой начинает формироваться гелевая матрица в высыхающей капле биологической жидкости. Также выявлена зависимость значения точки геля от формы и размера молекулы с учётом молекулярного докинга.

Работа выполнена при поддержке Министерства образования и науки РФ проект 15882011 «Математическое моделирование процессов самоорганизации в системах микро- и наночастиц».

\section{БИБЛИОГРАФИЧЕСКИЙ СПИСОК}

1. Stauffer D., Aharony A. Introduction to Percolation Theory. London: Taylor \& Francis, 1992. $181 \mathrm{pp}$.

2. Sahimi M. Application of Percolation Theory. London: Taylor \& Francis, 1994. 258 pp.

3. Ohira K., Sato M., Kohmoto M. Fluctuations in chemical gelation// Phys. Rev. E, 2007. Vol. 75, no. 4, 041402. 6 pp., arXiv: cond-mat/0510106 [cond-mat.soft].

4. Del Gado E., Fierro A., de Arcangelis L., Coniglio A. Slow dynamics in gelation phenomena: From chemical gels to colloidal glasses // Phys. Rev. E, 2004. Vol.69, no. 5, 051103. 9 pp., arXiv: cond-mat/0310776 [cond-mat.soft].

5. Plischke M., Vernon D. C., Joóc B. Model for gelation with explicit solvent effects: Structure and dynamics // Phys. Rev. E, 2003. Vol.67, no.1, 011401. 6 pp., arXiv: cond-mat/0207304 [cond-mat.soft].

6. Garboczi E. J., Snyder K. A., Douglas J. F., Thorpe M. F. Geometrical percolation threshold of overlapping ellipsoids // Phys. Rev. E, 1995. Vol. 52, no. 1. Pp. 819-828.

7. Yi. Y. B. Void percolation and conduction of overlapping ellipsoids // Phys. Rev. E, 2006. Vol. 74, no. 3, 031112. 6 pp.

8. Akagawa S., Odagaki T. Geometrical percolation of hard-core ellipsoids of revolution in the continuum // Phys. Rev. E, 2007. Vol.76, no. 5, 051402. 5 pp.

9. Ambrosetti G., Johner N. , Grimaldi C., Danani A., Ryser P. Percolative properties of hard oblate ellipsoids of revolution witha soft shell // Phys. Rev. E, 2008. Vol. 78, no. 6, 061126. 11 pp., arXiv: 0805.1181 [cond-mat.stat-mech].

10. Matsumoto M., Nishimura T. Mersenne twister: A 623-dimensionally equidistributed uniform pseudo-random number generator // ACM Trans. Model. Comput. Simul., 1998. Vol. 8, no. 1. Pp. 3-30.

11. Hoshen J., Kopelman R. Percolation and cluster distribution. I. Cluster multiple labeling technique and critical concentration algorithm // Phys. Rev. B, 1976. Vol.14, no. 8. Pp. 3438-3445.

12. Rubin F. The Lee path connection algorithm// IEEE Trans. Computers, 1974. Vol. C-23, no. 9. Pp. 907-914.

13. Тарасевич Ю. Ю. Перколяция: теория, приложения, алгоритмы. М.: Едиториал УРСС, 2002. 112 c. [Tarasevich Yu. Yu. Percolation. Theory, applications, algorithms. Moscow: Editorial URSS, 2002. 112 pp.]

14. Balberg I., Binenbaum N. Invariant properties of the percolation thresholds in the softcore-hard-core transition // Phys. Rev. A, 1987. Vol. 35, no. 12. Pp. 5174-5177.

15. Goncharuk A. I., Lebovka N. I., Lisetski L. N., Minenko S. S. Aggregation, percolation and phase transitions in nematic liquid crystal EBBA doped with carbon nanotubes // J. Phys. D: Appl. Phys., 2009. Vol. 42, no. 16, 165411. 8 pp. 
MSC: 60K35; 82B43, 82B26, 82B21

\section{PERCOLATION OF THE PROLATE ELLIPSOIDS OF ROTATION IN THE CONTINUUM}

\section{M. Buzmakova}

Astrakhan State University,

20 a, Tatishcheva st., Astrakhan, Russia, 414056.

E-mail: mariya_nazarova@mail.ru

Continuum percolation of the hard prolate ellipsoids of rotation with permeable shell has been investigated. It is the model of phase transition sol-gel. Ellipsoids are located in the cube randomly. For each set of parameters 100 tests are spent. For each test the finding of the percolation cluster is the main task. The fraction of the packing for which the probability of the percolation cluster appearance is equal 0.5, is called a percolation threshold. Value of the percolation threshold corresponds to the gel point. Dependence of value of the percolation threshold on thickness of permeable shell and aspect ratio has been obtained. In addition to the percolation threshold the other characteristics of the model have been obtained, such as: the size distribution of clusters, the average cluster size, the strength and the fractal dimension of the percolation cluster, the average value and the distribution of neighbors of an element, the critical exponents.

Key words: percolation, gelation, phase transitions.

Original article submitted 09/VI/2012; revision submitted 25/IX/2012.

Mariya M. Buzmakova, Assistant, Dept. of Applied Mathematics \& Computer Science. 